\title{
A Dynamic and Heuristic Phase Balancing Method for LV Feeders
}

\author{
Samad Taghipour Boroujeni, ${ }^{1}$ Mohammad Mardaneh, ${ }^{2}$ and Zhale Hashemi ${ }^{3}$ \\ ${ }^{1}$ Department of Engineering, Shahrekord University, Shahrekord 34141-88186, Iran \\ ${ }^{2}$ Department of Electrical and Electronic Engineering, Shiraz University of Technology, Shiraz 71555-313, Iran \\ ${ }^{3}$ Department of Electrical Engineering, Islamic Azad University, Zarghan Branch, Zarghan, Iran \\ Correspondence should be addressed to Mohammad Mardaneh; mardaneh@sutech.ac.ir
}

Received 10 November 2015; Revised 31 March 2016; Accepted 13 April 2016

Academic Editor: Christian W. Dawson

Copyright (c) 2016 Samad Taghipour Boroujeni et al. This is an open access article distributed under the Creative Commons Attribution License, which permits unrestricted use, distribution, and reproduction in any medium, provided the original work is properly cited.

\begin{abstract}
Due to the single-phase loads and their stochastic behavior, the current in the distribution feeders is not balanced. In addition, the single-phase loads are located in different positions along the LV feeders. So the amount of the unbalanced load and its location affect the feeder losses. An unbalanced load causes the feeder losses and the voltage drop. Because of time-varying behavior of the single-phase loads, phase balancing is a dynamic and combinatorial problem. In this research, a heuristic and dynamic solution for the phase balancing of the LV feeders is proposed. In this method, it is supposed that the loads' tie could be connected to all phases through a three-phase switch. The aim of the proposed method is to make the feeder conditions as balanced as possible. The amount and the location of single-phase loads are considered in the proposed phase balancing method. Since the proposed method needs no communication interface or no remote controller, it is inexpensive, simple, practical, and robust. Applying this method provides a distributed and dynamic phase balancing control. In addition, the feasibility of reducing the used switches is investigated. The ability of the proposed method in the phase balancing of the LV feeders is approved by carrying out some simulations.
\end{abstract}

\section{Introduction}

Nowadays, fossil fuels, the main source of our world energy, have been diminished day by day. In addition, the environmental pollution and the global warming concerns cause the increase in the importance of energy saving and loss reduction. So a lot of researches have been devoted to enhancing the efficiency of the energy converter machines. The most popular shape of energy, electrical energy, is mainly produced in the power plants, transmitted through the electrical networks, and consumed in the load points. Therefore, the electrical network efficiency has a greater role in the energy saving and loss reduction. Electrical networks include transmission and distribution systems. As the transmission voltage is higher than the distribution voltage, the distribution system losses are greater.

The phase balancing results in benefits such as loss reduction, voltage profile improvement, and system reliability enhancement. At LV feeders, single-phase loads make unbalanced conditions and increase losses in the LV feeders. These loads are distributed along the feeder and have different and stochastic behaviors. All of these aspects of the singlephase loads make distribution phase balancing more difficult and complicated. In fact phase balancing is a dynamic combinatorial problem.

The distribution load balancing has been considered in many researches. These researches can be divided into two main groups. The first group deals with the network reconfiguration through some switches [1-12]. These methods try to distribute the power demanded by one geographical zone among different feeders equally. In the second group, it is desired to distribute the power of one feeder among its different phases, equally. In addition, against profound and strong researches on load balancing [1-12], there are few papers on phase balancing [13-15]. In [13, 14], medium voltage phase balancing is addressed by choosing the best connection for the transformer nodes. 
Traditionally, phase balancing is done at periodical intervals, manually, after some field measurements by the utilities $[15,16]$, which is mentioned here as the Static phase balancing (SPB). Although the SPB is a stable method, due to the stochastic and time-varying characteristics of the singlephase loads, it is not an efficient method in the loss reduction. On the other hand, the dynamic phase balancing (DPB) is a more effective method in the loss reduction. However, the $\mathrm{DPB}$ method is an expensive method and enough attention should be paid to its instability [16]. The DPB of the LV feeders is addressed in $[15,16]$ using a central remote controller and communication system.

DPB can be implemented easily in the distribution network equipped with an automated system $[15,16]$. Although advances in power electronic devices, microcontroller processors, and communication systems make it easy to apply automation systems in the distribution networks, in many countries it needs a long time to have such systems, completely.

Since single-phase loads in LV networks are distributed along the feeder, their location is an important and effective parameter on the feeder losses, as well as their amount. In comparison with an unbalanced load in the first sections of LV feeder, the same unbalanced load in the end points of the considered feeder results in more losses. The impact of singlephase load locations is not considered in $[15,16]$. In addition, the proposed methods in $[15,16]$ need an automated system with fuzzy or neural remote controllers, which make DPB system expensive and complicated.

In this research a heuristic and dynamic solution for phase balancing of LV feeders is proposed. In this method, it is supposed that customers' ties are switchable between different phases through a three-phase switch. The threephase switches are controlled by some distributed local controllers. These local controllers only need locally measured signals without any information about the other loads and branches which are far from the controller installing point. Therefore, the communication interface can be avoided. The proposed method tries to make the current in each section of the LV feeder as balanced as possible. In the proposed method amount and location of single-phase loads are considered inherently. Although some three-phase switches are used in the proposed method, it is inexpensive, simple, and practical. In some cases it may happen that the benefit of the loss reduction cannot overcome the cost of the installed switches. Therefore, a trade-off between the loss reduction and the number of the used switches should be done. So the Genetic Algorithm is applied to find the optimum number of switches as well as their locations. The proposed method does not need any remote controller and communication interface. So it is a fault tolerant method which provides a distributed and dynamic phase balancing control.

Hereafter phase balancing problem is represented in Section 2. The proposed algorithm is described in Section 3. In addition, the feasibility of reducing the number of the switches is investigated in Section 4. Simulation results are reported in Section 5. These results show the ability and the efficiency of the proposed method in phase balancing of the LV feeders.

\section{Problem Definition}

For the sake of simplicity, loads are modeled as the constant currents in 4-wire, 3-phase distribution systems. Also KCL law is used to obtain branches current instead of the forwardbackward load flow method. These assumptions' consideration does not have effect on the generality of the phase balancing problem. Total loss of a LV feeder is as follows:

$$
P_{\text {loss }}=\sum_{x \in S} \sum_{k=1}^{m} \underset{(x)}{\stackrel{(k)}{r}} \cdot\left(\begin{array}{c}
(k) \\
I \\
(x)
\end{array}\right)^{2},
$$

where $S$ contains phases $a, b$, and $c$ and the neutral wire, $\underset{(x)}{I}$ and $\underset{(x)}{r}$ are the current and the resistance of the phase " $x$ " of the branch " $k$," respectively, and " $m$ " is number of the feeder branches.

In the presented paper, the radial LV feeder is studied as a graph. In the graph theory, the nodes are connected via some branches. Some concepts and definitions of the graph theory which are used in this research are as follows.

(i) Branch. A line section which connects two nodes together is defined as the branch. A branch contains at least two-wire branch (1-phase and 1-neutral wire).

(ii) Main Node. Main node is the nearest node of the LV feeder to the transformer. It is located at the beginning of the feeder. This node connects LV and MV networks together.

(iii) End Node. The end nodes are the deepest nodes in the feeder. From these nodes no branch is initiated.

(iv) Node Path. In a radial graph each node can be connected to the main node only in one path, called here the node path. The node path is a sequence of branches or nodes, which connect the considered node to the main node.

(v) Upstream and Downstream Nodes. Considering two nodes ( $A$ and $B$ ) of a branch, the node that is nearer to the main node is the upstream node of the other one. Similarly the latter one is the downstream node of the former. In other words, node $A$ is upstream to node $B$ (or node $B$ is downstream to node $A$ ), if the path sequence of node $A$ is a subset of the path sequence of node $B$. Since the LV feeder graph is considered radial, each node has only one upstream node, except the main node. In addition, except the end nodes that have no downstream node, the other nodes have at least one downstream node.

(vi) Node Ranking. The criterion for the ranking is the position of nodes in the node paths. In the other words, the rank of an upstream node is more than the rank of its downstream nodes. In the node ranking, different nodes could have the same rank.

(vii) Upstream and Downstream Branches. Each node is connected to other nodes through some branches. For each node, the upstream branch is the branch which connects the considered node to its upstream node (or other nodes with a higher rank). Similarly the downstream branch is defined as the branch which connects the considered node 


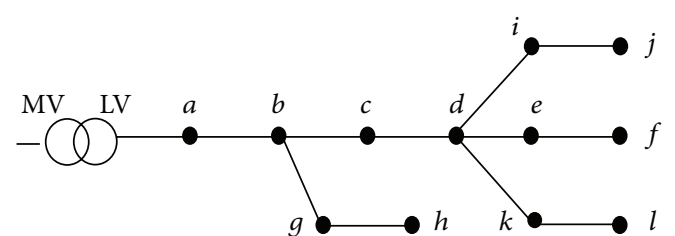

(a)

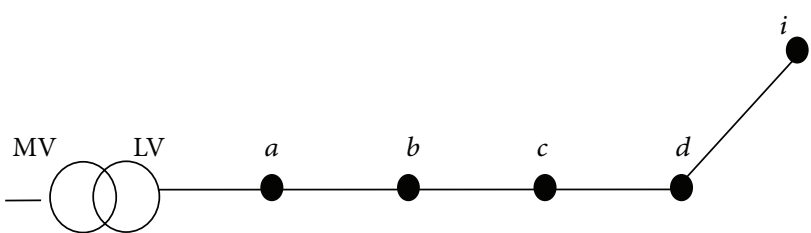

(b)

FIgURE 1: (a): The considered 12-node feeder graph. (b) A subgraph of (a).

to one of the downstream nodes (or other nodes with lower rank). Since the LV feeder graph is radial, each node has only one upstream branch except the main node that has no upstream branch. In addition, except end nodes that have no downstream branches, other nodes have at least one downstream branch.

(viii) Subgraph. A subgraph is a continuous graph whose nodes/branches are subsets of the main graph nodes/ branches.

(ix) An Example. In order to make the above definitions more clear, an example is presented. Figure 1(a) shows a small radial LV feeder with 12 nodes and 11 branches. Node " $a$ " is the main node and nodes $\{g, j, f, l\}$ are the end nodes. The path of node " $k$ " is $\{a, b, c, d\}$. The upstream and downstream nodes of node " $i$ " are " $d$ " and " $j$," respectively. Branch " $c d$ " is the upstream branch of node " $d$ " and its downstream branches are $\{d i, d e, d k\}$. The nodes ranking are as follows: $\operatorname{rank}(a)=1$, $\operatorname{rank}(b)=2, \operatorname{rank}(c)=\operatorname{rank}(g)=3, \operatorname{rank}(h)=\operatorname{rank}(d)=4$, $\operatorname{rank}(e)=\operatorname{rank}(i)=\operatorname{rank}(k)=5, \operatorname{and} \operatorname{rank}(j)=\operatorname{rank}(f)=$ $\operatorname{rank}(l)=6$. Also the path of node " $i$," shown in Figure 1(b), is a subgraph of the feeder graph of Figure 1(a).

\section{The Proposed Heuristic Algorithm}

The proposed heuristic method tries to make the phases current in each branch as balanced as possible. This condition should be very close to the optimal phase configuration with the lowest losses. For this purpose, it is supposed that the load of each node can be switched to different phases from a local controller installed on the node. A local controller installed on the node as determine the energizing phase. The input signals of the local controller are the measured load currents of the node as well as phase currents of the downstream branches of the considered node. Algorithm of phase selecting is as follows. This algorithm has to be carried out in the predefined intervals.

(1) The phase assigning is carried out step by step, from the lowest ranked node (end nodes) to the highest ranked node (main node). Nodes with the same rank are analyzed in one step.

(2) The currents of the downstream branches of one node are measured and obtained from

$$
\underset{(x)}{\mathrm{DC}}=\sum_{j \in J} \underset{(x)}{I_{L}}
$$

where $\underset{(x)}{\mathrm{DC}}$ is the sum of currents in phase " $x$ " in downstream branches of node " $k, \stackrel{j}{I}_{L}$ is the load current of node " $j$ " in phase " $x$ ", $x \in\{a, b, c\}, J$ includes all the nodes which their paths contain node $k$, and $\underset{(x)}{\mathrm{DC}}$ can be obtained from

$$
\underset{(x)}{\mathrm{DC}}=\underset{(x)}{\stackrel{(k)}{\mathrm{U}} \mathrm{C}}-\underset{(x)}{I_{L}}
$$

where ÜC is the phase " $x$ " current in the upstream branch of $(x)$ node " $k$ ". Obtaining $\underset{(x)}{(k)}$ from (3) is easier and more practical than using (2).

Note. For the end nodes $\underset{(x)}{\mathrm{DC}}$ is considered zero.

(3) Based on the obtained value of $\underset{(x)}{\mathrm{DC}}$, the load of node " $k$ " is connected to one of the existing phases at node " $k$ " such that its upstream branch current is made as balanced as possible. In order to find the best phase selection for the load at node " $k$," a direct search is applied such that (4) is minimized. Consider

$$
\sum_{x \in\{a, b, c\}}\left|\left(I_{\mathrm{av}}-\underset{(x)}{\mathrm{DC}}-\underset{(x)}{\stackrel{(k)}{I}}\right)\right|
$$

where

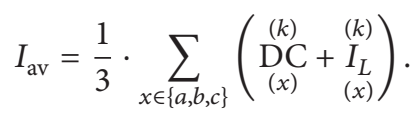

The above steps are done until the connecting phase at all nodes are determined. Since single-phase loads are varying in time, repeating the above algorithm in predefined interval is necessary to hold the LV feeder in optimum condition at all times.

\section{Optimization}

As mentioned, installing three-phase switches in all load connections, and controlling them from a remote or local controller, is an expensive method. Here, it is supposed to 


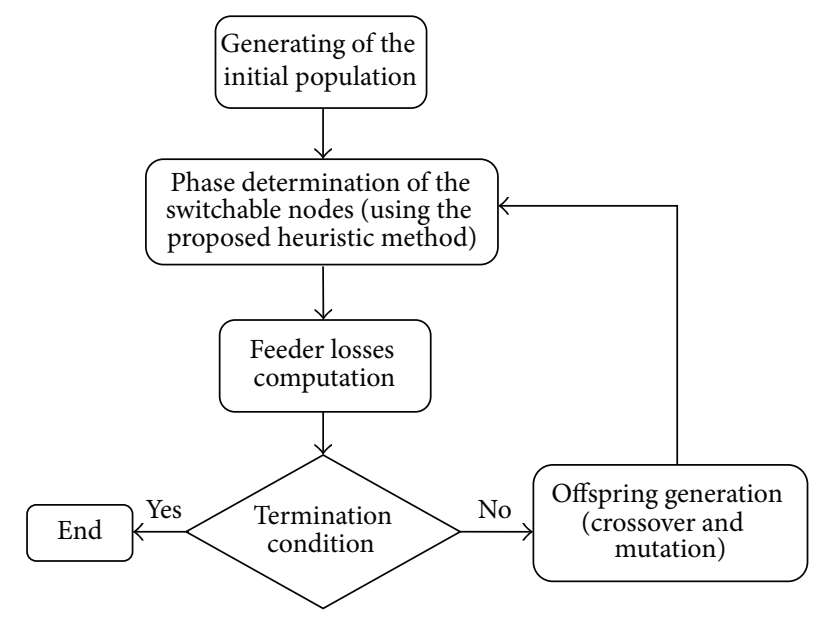

Figure 2: The flowchart of the used GA.

\begin{tabular}{rl|l|l|l|l|} 
Node number & \multicolumn{1}{c}{2} & \multicolumn{3}{c|}{$n-1$} & \multicolumn{1}{c}{$n$} \\
\cline { 2 - 6 } Switchable nodes & & & $\cdots$ & & \\
\cline { 2 - 7 } Fixed nodes' phases & & & $\cdots$ & & \\
\cline { 2 - 6 } & & &
\end{tabular}

FIGURE 3: The chromosome structure used in GA.

have fixed ties for some nodes and the other nodes can be switched to different phases. The main goal of this section is to find the minimum number of installed switches and their location. Also other nodes phases have to be determined to achieve the lowest losses. For this purpose a Genetic Algorithm (GA) is applied as the optimization tool. Objective function which is going to be minimized is feeder losses. In order to decrease computation time of GA, especially in feeders with a large number of nodes, the proposed heuristic method is combined with GA and referred to here as Modified Genetic Algorithm (MGA).

4.1. GA Description. The flowchart of the used GA is presented in Figure 2. This flowchart is carried out for all load conditions. The chromosome in this algorithm is as in Figure 3. The first row of this chromosome is considered for the switchable nodes. It is $\mathbf{1}$ if a three-phase switch is installed in that node, otherwise it is $\mathbf{0}$. The second row shows phase connection of the fixed nodes. For achieving this purpose $\{1,2,3\}$ indicates the phases $\{a, b, c\}$.

4.2. MGA Description. In comparison with GA, the search space is modified in MGA. This results in a shorter computation time and a better convergence in MGA. The flowchart of the used MGA is shown in Figure 4. The considered chromosome in MGA is as in Figure 5. Similar to the first row of the considered chromosome in GA, switchable nodes are modeled with $\mathbf{1}$ and the other nodes are $\mathbf{0 .}$

In the MGA, the GA operators such as the crossover and the mutation are used to generate new offspring. In addition, the proposed heuristic method is used to determine the connecting phase at all nodes. In order to determine phase connection of the nodes with the fixed ties, average daily value

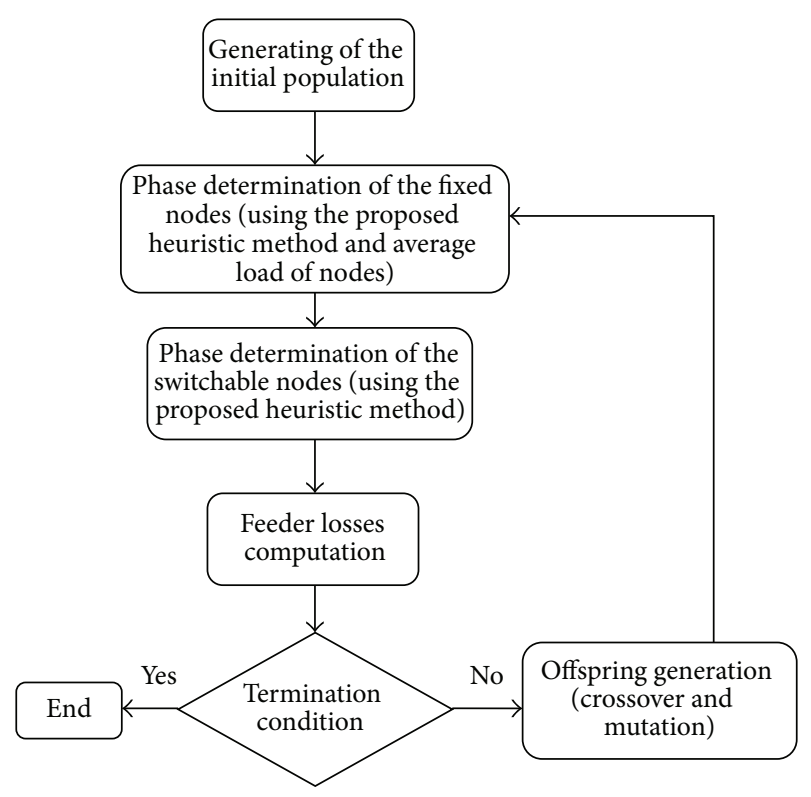

FIgURE 4: The flowchart of the used MGA.

\begin{tabular}{r|c|c|c|c|c|} 
Node number & 1 & \multicolumn{1}{c}{2} & \multicolumn{1}{c}{$n-1$} & $n$ \\
\cline { 2 - 6 } Switchable nodes & & & $\cdots$ & & \\
\cline { 2 - 6 }
\end{tabular}

FIgURE 5: The chromosome structure used in MGA.

of load current at these nodes is considered in the proposed heuristic method.

\section{Simulation Results and Comparison}

In this section the simulation results are used to investigate capability of the proposed heuristic method in phase balancing. For this purpose real measured data reported in [15] for 15-node and 45-node radial feeders are used. These data are represented in Tables 1 and 2. Configuration of the 15-node feeder is as in Figure 6. 45-node feeder configuration is the same as 15-node feeder (all the nodes are located in one line). In Table 1, 15-node feeder loads current is reported for three different conditions. Branches spans are not reported in [15]. The aim of [15] has been only to reach balanced currents in the main node branches of the feeder. Loads are connected to different phases through a three-phase switch. Static phase balancing (SPB) is carried out as second column in this table [15]. Of course branches spans (and their impedances) have a great impact on feeder losses. Last columns of Tables 1 and 2 show the considered branches spans.

Table 3 shows the obtained switches states from different methods for phase balancing in different conditions. In this table $\mathrm{NN}$ and $\mathrm{HE}$ are the proposed neural network and heuristic methods in [15], respectively. HM refers to the proposed heuristic method in this paper. In Table 4 the 15node feeder losses and its first branch (node "1" to node "2") phase currents are reported. It is obvious that HE [15] is more effective than NN [15]. The best cases are in bold font in Table 4. Average feeder losses over different conditions for 
TABLE 1: The considered 15-node feeder loads and branches data.

\begin{tabular}{lcccccc}
\hline Node & SPB & Load data 1 (A) & Load data $2(\mathrm{~A})$ & Load data $3(\mathrm{~A})$ & \multicolumn{2}{c}{$\begin{array}{c}\text { Branches data } \\
\text { End node }\end{array}$} \\
\hline 1 & 1 & 94.06 & 40.16 & 1.51 & 1 & 2 \\
Length $(\mathrm{m})$
\end{tabular}

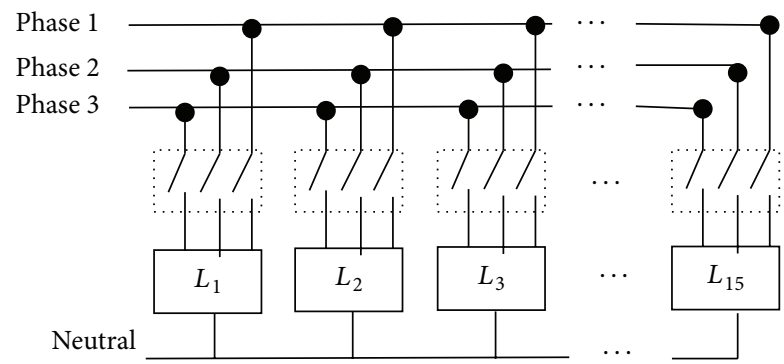

FIgURE 6: The considered 15-node feeder configuration.

different phase balancing, SPB, NN [15], HE [15], and HM are $10.8 \mathrm{~kW}, 14 \mathrm{~kW}, 10.8 \mathrm{~kW}$, and $9.44 \mathrm{~kW}$, respectively. Although HE [15] can balance the first branch current better than $\mathrm{HM}$ for conditions 2 and 3, its loss is more than that which HM has. The reason is that the unbalanced load position is effective on the feeder losses as well as its value. This fact is not considered in $[15,16]$. Table 5 shows the obtained results for phase balancing of 45-node feeder with different methods. Switching table of these methods for 45-node feeder is reported in columns $2-5$ of Table 2.

5.1. Optimization Results. The feasibility of decreasing the number of the used three-phase switches and providing an inexpensive phase balancing system is investigated with GA and MGA. Obtained results of feeder losses for different number of used switches are reported in Figure 7. The obtained results from GA and MGA are very close to each other, while MGA is much faster than GA. Mean computation time for GA and MGA is 1.35 seconds and 98 seconds, respectively.

Another important aspect is that the 15-node feeder average losses are $9.44 \mathrm{~kW}$ when three-phase switches are installed in all 15 nodes, while based on Figure 7 decreasing

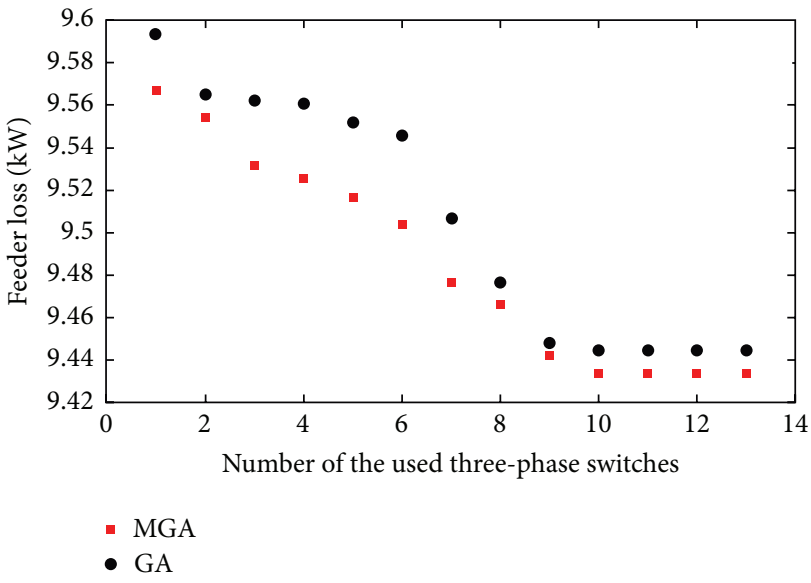

FIGURE 7: Average feeder loss versus number of installed switches in 15-node feeder over different condition of Table 1.

switches does not increase feeder losses too much. Selecting the best number of three-phase switches depends on energy cost for utilities.

\section{Conclusion}

In this paper a heuristic method is proposed for phase balancing of LV feeders. It applies three-phase switches in some nodes. These switches are controlled locally without any information about the other branches and loads current or the other switches status. These reduce the cost of the phase balancing system due to removing central remote controller and related communication systems. The ability of the proposed method is compared with other phase balancing methods. In comparison with the other dynamic 
TABLE 2: The considered 45-node feeder loads and branches data.

\begin{tabular}{|c|c|c|c|c|c|c|c|c|}
\hline \multirow{2}{*}{ Node } & \multirow{2}{*}{ Load (A) } & \multicolumn{4}{|c|}{ Switches state } & \multicolumn{3}{|c|}{ Branches data } \\
\hline & & SPB & $\mathrm{NN}$ [15] & HE [15] & FHM & First node & End node & Length ( $\mathrm{m})$ \\
\hline 1 & 40.16 & 1 & 1 & 1 & 2 & 1 & 2 & 20 \\
\hline 2 & 92.61 & 2 & 2 & 1 & 3 & 2 & 3 & 25 \\
\hline 3 & 90.77 & 3 & 3 & 2 & 1 & 3 & 4 & 15 \\
\hline 4 & 40.61 & 1 & 1 & 3 & 1 & 4 & 5 & 25 \\
\hline 5 & 88.47 & 2 & 3 & 3 & 2 & 5 & 6 & 25 \\
\hline 6 & 5.73 & 3 & 1 & 1 & 2 & 6 & 7 & 20 \\
\hline 7 & 34.93 & 1 & 3 & 3 & 1 & 7 & 8 & 15 \\
\hline 8 & 80.5 & 2 & 1 & 2 & 3 & 8 & 9 & 20 \\
\hline 9 & 0.97 & 3 & 2 & 2 & 3 & 9 & 10 & 15 \\
\hline 10 & 13.75 & 1 & 1 & 1 & 1 & 10 & 11 & 25 \\
\hline 11 & 20.07 & 2 & 3 & 2 & 3 & 11 & 12 & 20 \\
\hline 12 & 19.67 & 3 & 2 & 2 & 1 & 12 & 13 & 20 \\
\hline 13 & 59.77 & 1 & 3 & 1 & 2 & 13 & 14 & 25 \\
\hline 14 & 26.94 & 2 & 2 & 3 & 3 & 14 & 15 & 15 \\
\hline 15 & 19.68 & 3 & 2 & 3 & 3 & 15 & 16 & 20 \\
\hline 16 & 1.51 & 1 & 1 & 1 & 3 & 16 & 17 & 20 \\
\hline 17 & 73.93 & 2 & 2 & 2 & 1 & 17 & 18 & 25 \\
\hline 18 & 44.06 & 3 & 3 & 3 & 3 & 18 & 19 & 15 \\
\hline 19 & 92.24 & 1 & 1 & 2 & 2 & 19 & 20 & 25 \\
\hline 20 & 46.13 & 2 & 1 & 1 & 3 & 20 & 21 & 25 \\
\hline 21 & 41.44 & 3 & 2 & 2 & 2 & 21 & 22 & 20 \\
\hline 22 & 83.77 & 1 & 3 & 3 & 1 & 22 & 23 & 15 \\
\hline 23 & 51.99 & 2 & 1 & 1 & 3 & 23 & 24 & 20 \\
\hline 24 & 20.06 & 3 & 3 & 2 & 3 & 24 & 25 & 15 \\
\hline 25 & 66.54 & 1 & 2 & 3 & 1 & 25 & 26 & 25 \\
\hline 26 & 82.97 & 2 & 2 & 1 & 2 & 26 & 27 & 20 \\
\hline 27 & 1.94 & 3 & 3 & 3 & 2 & 27 & 28 & 20 \\
\hline 28 & 67.44 & 1 & 1 & 3 & 3 & 28 & 29 & 25 \\
\hline 29 & 37.56 & 2 & 1 & 2 & 3 & 29 & 30 & 15 \\
\hline 30 & 82.34 & 3 & 1 & 1 & 2 & 30 & 31 & 20 \\
\hline 31 & 94.06 & 1 & 1 & 1 & 1 & 31 & 32 & 20 \\
\hline 32 & 22.88 & 2 & 2 & 2 & 2 & 32 & 33 & 25 \\
\hline 33 & 60.07 & 3 & 1 & 1 & 3 & 33 & 34 & 15 \\
\hline 34 & 48.11 & 1 & 3 & 3 & 2 & 34 & 35 & 25 \\
\hline 35 & 88.23 & 2 & 1 & 3 & 1 & 35 & 36 & 25 \\
\hline 36 & 75.44 & 3 & 1 & 3 & 3 & 36 & 37 & 20 \\
\hline 37 & 45.19 & 1 & 2 & 2 & 1 & 37 & 38 & 15 \\
\hline 38 & 1.83 & 2 & 3 & 3 & 1 & 38 & 39 & 20 \\
\hline 39 & 81.31 & 3 & 2 & 3 & 2 & 39 & 40 & 15 \\
\hline 40 & 60.92 & 1 & 1 & 1 & 3 & 40 & 41 & 25 \\
\hline 41 & 78.4 & 2 & 3 & 2 & 1 & 41 & 42 & 20 \\
\hline 42 & 91.25 & 3 & 2 & 1 & 2 & 42 & 43 & 20 \\
\hline 43 & 73.08 & 1 & 2 & 2 & 3 & 43 & 44 & 25 \\
\hline 44 & 17.45 & 2 & 2 & 2 & 2 & 44 & 45 & 15 \\
\hline 45 & 44.02 & 3 & 1 & 1 & 1 & & $r=0.2 \Omega / \mathrm{kn}$ & \\
\hline
\end{tabular}


TABLE 3: Switching table of 15-node feeder for different methods of phase balancing.

\begin{tabular}{|c|c|c|c|c|c|c|c|c|c|}
\hline \multirow{2}{*}{ Node } & \multicolumn{3}{|c|}{ Condition 1} & \multicolumn{3}{|c|}{ Condition 2} & \multicolumn{3}{|c|}{ Condition 3} \\
\hline & NN [15] & HE [15] & HM & NN [15] & HE [15] & $\mathrm{HM}$ & NN [15] & HE [15] & $\mathrm{HM}$ \\
\hline 1 & 1 & 1 & 1 & 1 & 1 & 3 & 1 & 1 & 1 \\
\hline 2 & 2 & 2 & 2 & 2 & 1 & 1 & 2 & 2 & 2 \\
\hline 3 & 1 & 1 & 3 & 3 & 2 & 2 & 3 & 3 & 1 \\
\hline 4 & 3 & 3 & 2 & 1 & 3 & 1 & 1 & 2 & 3 \\
\hline 5 & 1 & 3 & 1 & 3 & 3 & 3 & 1 & 1 & 1 \\
\hline 6 & 1 & 3 & 3 & 1 & 1 & 3 & 2 & 2 & 3 \\
\hline 7 & 2 & 2 & 1 & 3 & 3 & 1 & 3 & 3 & 2 \\
\hline 8 & 3 & 3 & 1 & 1 & 2 & 2 & 1 & 1 & 1 \\
\hline 9 & 2 & 3 & 2 & 2 & 2 & 2 & 3 & 2 & 1 \\
\hline 10 & 1 & 1 & 3 & 1 & 1 & 1 & 2 & 3 & 3 \\
\hline 11 & 3 & 2 & 1 & 3 & 2 & 2 & 2 & 1 & 2 \\
\hline 12 & 2 & 1 & 2 & 2 & 2 & 1 & 3 & 3 & 2 \\
\hline 13 & 2 & 2 & 3 & 3 & 1 & 3 & 1 & 3 & 3 \\
\hline 14 & 2 & 2 & 2 & 2 & 3 & 2 & 1 & 2 & 2 \\
\hline 15 & 1 & 1 & 1 & 2 & 3 & 1 & 1 & 1 & 1 \\
\hline
\end{tabular}

TABLE 4: Different phase balancing results for 15-node feeder.

\begin{tabular}{|c|c|c|c|c|c|c|}
\hline & & $I_{a}(\mathrm{~A})$ & $I_{b}(\mathrm{~A})$ & $I_{c}(\mathrm{~A})$ & $\Delta I_{\max }(\mathrm{A})$ & Total feeder loss $(\mathrm{kW})$ \\
\hline \multirow{4}{*}{ Condition 1} & SPB & 321.36 & 208.79 & 352.09 & 143.30 & 14.44 \\
\hline & NN [15] & 422.74 & 331.16 & 128.34 & 294.4 & 18.05 \\
\hline & HE [15] & 350.32 & 237 & 249.92 & 113.32 & 14.07 \\
\hline & HM & 351.73 & 261 & 269.51 & 90.73 & 12.33 \\
\hline \multirow{4}{*}{ Condition 2} & $\mathrm{SPB}$ & 189.22 & 308.59 & 136.82 & 171.77 & 5.98 \\
\hline & NN [15] & 180.75 & 159.87 & 294.01 & 134.14 & 6.43 \\
\hline & HE [15] & 212.02 & 211.98 & 210.63 & 1.39 & 5.26 \\
\hline & HM & 221.25 & 219.25 & 194.3 & 27.12 & 4.83 \\
\hline \multirow{4}{*}{ Condition 3} & SPB & 311.50 & 292.58 & 189.84 & 121.66 & 12 \\
\hline & $\mathrm{NN}$ [15] & 379.21 & 264.88 & 149.83 & 229.38 & 17.55 \\
\hline & HE [15] & 264.94 & 265.23 & 263.75 & 1.48 & 12.99 \\
\hline & HM & 244.58 & 280.17 & 267.66 & 34.08 & 11.16 \\
\hline
\end{tabular}

TABLE 5: Different phase balancing results for 45-node feeder.

\begin{tabular}{lcccc}
\hline & SPB & NN [15] & HE [15] & HM \\
\hline$I_{a}(\mathrm{~A})$ & 822.08 & 982.7 & 827.28 & 775.7 \\
$I_{b}(\mathrm{~A})$ & 809.96 & 755.91 & 714.21 & 756.06 \\
$I_{c}(\mathrm{~A})$ & 678.75 & 572.18 & 769.3 & 779.03 \\
$\Delta I_{\max }(\mathrm{A})$ & 143.33 & 410.52 & 113.07 & $\mathbf{3 . 3 3}$ \\
Total feeder loss $(\mathrm{kW})$ & 118.4 & 150 & 119 & $\mathbf{1 1 4 . 2 6}$ \\
\hline
\end{tabular}

phase balancing methods $[14,15]$, the location of the unbalanced load is considered in the proposed heuristic method. Simulation results in Table 4 and Figure 7 show merit of the proposed method over the previous ones. Also possibility of using the proposed method in the optimization of the feeder losses with a few number of three-phase switches is investigated. It is shown that using the proposed method decreases computation time of GA convergence considerably.
This fact is important especially in the feeders with a lot of nodes.

\section{Competing Interests}

The authors declare that they have no competing interests.

\section{References}

[1] M. E. Baranand and F. F. Wu, "Network reconfiguration in distribution system for loss reduction and load balancing," IEEE Transactions on Power Delivery, vol. 4, no. 2, pp. 1401-1407, 1989.

[2] M. A. Kashem, G. B. Jasmon, and V. Ganapathy, "New approach of distribution system reconfiguration for loss minimization," International Journal of Electrical Power and Energy System, vol. 22, no. 4, pp. 269-276, 2000.

[3] R. Taleski and D. Rajičić, "Distribution network reconfiguration for energy loss reduction," IEEE Transactions on Power Systems, vol. 12, no. 1, pp. 398-406, 1997. 
[4] T. P. Wagner, A. Y. Chikhani, and R. Hackam, "Feeder reconfiguration for loss reduction: an application of distribution automation," IEEE Transactions on Power Delivery, vol. 6, no. 4, pp. 1922-1933, 1991.

[5] M. A. Kashem, V. Ganapathy, and G. B. Jasmon, "Network reconfiguration for load balancing in distribution networks," IEE Proceedings: Generation, Transmission and Distribution, vol. 146, no. 6, pp. 563-567, 1999.

[6] M. A. Kashem and V. Ganapathy, "Three-phase load balancing in distribution systems using index measurement technique," International Journal of Electrical Power and Energy Systems, vol. 24, no. 1, pp. 31-40, 2002.

[7] D. Das, "Reconfiguration of distribution system using fuzzy multi-objective approach," International Journal of Electrical Power \& Energy Systems, vol. 28, no. 5, pp. 331-338, 2006.

[8] C.-H. Lin, "Distribution network reconfiguration for load balancing with a coloured Petri net algorithm," IEE Proceedings: Communications, vol. 150, no. 3, pp. 317-324, 2003.

[9] S. K. Goswami and S. K. Basu, "A new algorithm for the reconfiguration of distribution feeders for loss minimization," IEEE Transactions on Power Delivery, vol. 7, no. 3, pp. 1484-1491, 1992.

[10] C. S. Chen and M. Y. Cho, "Energy loss reduction by critical switches," IEEE Transactions on Power Delivery, vol. 8, no. 3, pp. 1246-1253, 1993.

[11] C.-C. Liu, S. J. Lee, and K. Vu, "Loss minimization of distribution feeders: optimality and algorithms," IEEE Transactions on Power Delivery, vol. 4, no. 2, pp. 1281-1289, 1992.

[12] H. Salazar, R. Gallego, and R. Romero, "Artificial neural networks and clustering techniques applied in the reconfiguration of distribution systems," IEEE Transactions on Power Delivery, vol. 21, no. 3, pp. 1735-1742, 2006.

[13] T.-H. Chen and J.-T. Cherng, "Optimal phase arrangement of distribution transformers connected to a primary feeder for system unbalance improvement and loss reduction using a genetic algorithm," in Proceedings of the IEEE International Conference on Power Industry Computer Applications (PICA '99), pp. 145-151, Santa Clara, Calif, USA, May 1999.

[14] A. Augugliaro, L. Dusonchet, M. G. Ippolito, and E. R. Sanseverino, "Minimum losses reconfiguration of MV distribution networks through local control of tie-switches," IEEE Transactions on Power Delivery, vol. 18, no. 3, pp. 762-771, 2003.

[15] M. W. Siti, D. V. Nicolae, A. A. Jimoh, and A. Ukil, "Reconfiguration and load balancing in the LV and MV distribution networks for optimal performance," IEEE Transactions on Power Delivery, vol. 22, no. 4, pp. 2534-2540, 2007.

[16] A. Ukil and W. Siti, "Feeder load balancing using fuzzy logic and combinatorial optimization-based implementation," Electric Power Systems Research, vol. 78, no. 11, pp. 1922-1932, 2008. 

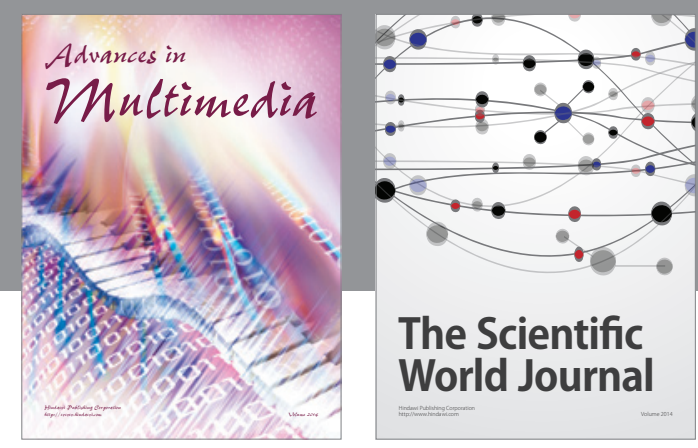

The Scientific World Journal
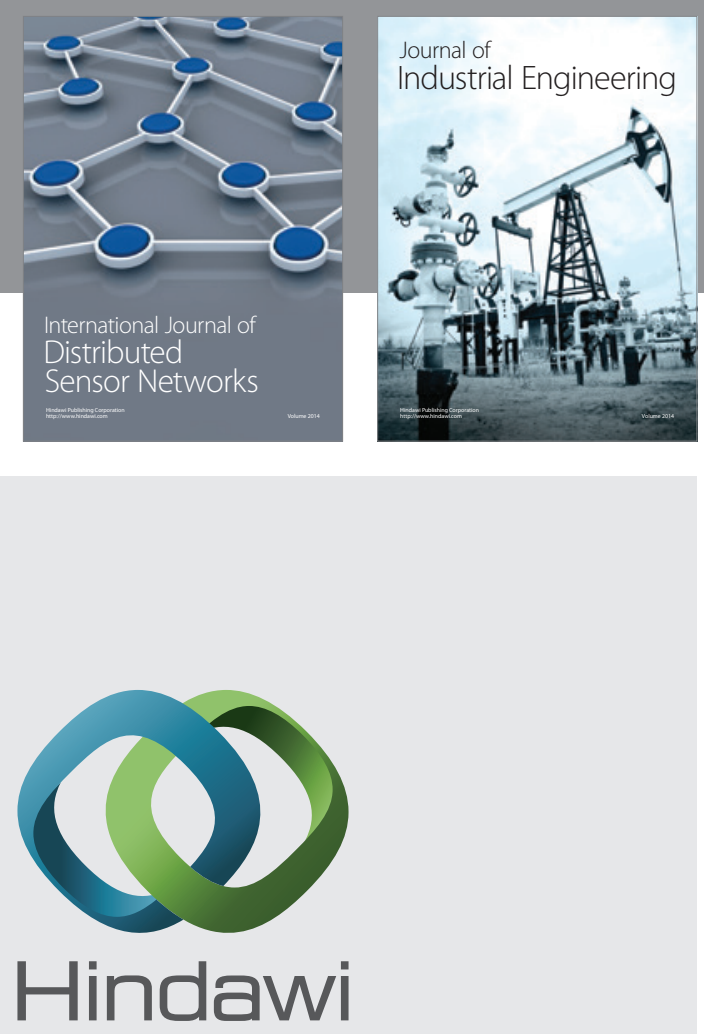

Submit your manuscripts at

http://www.hindawi.com

\section{Computer Networks} and Communications
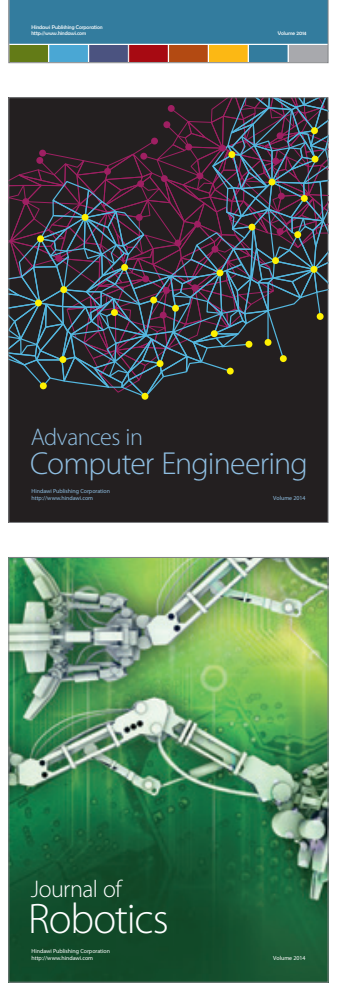
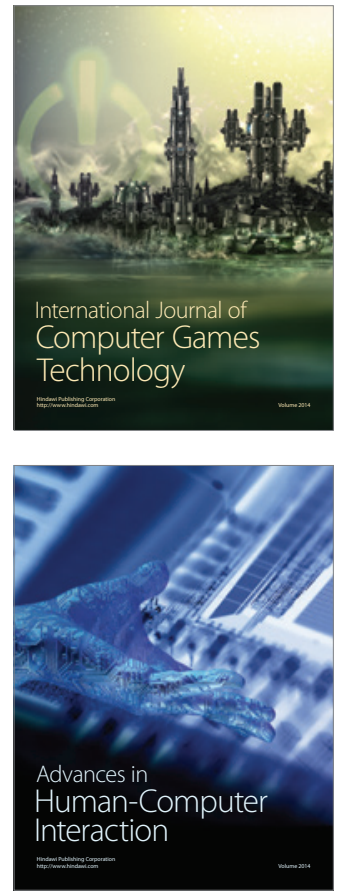
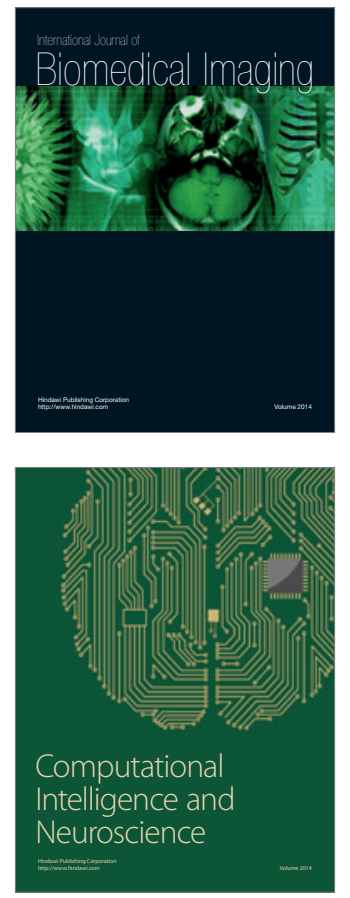
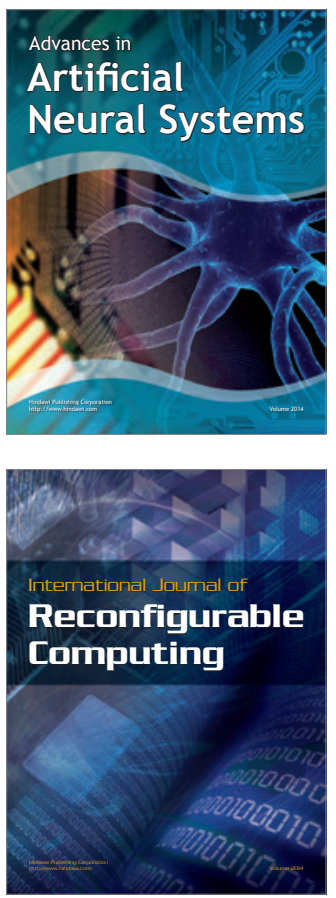
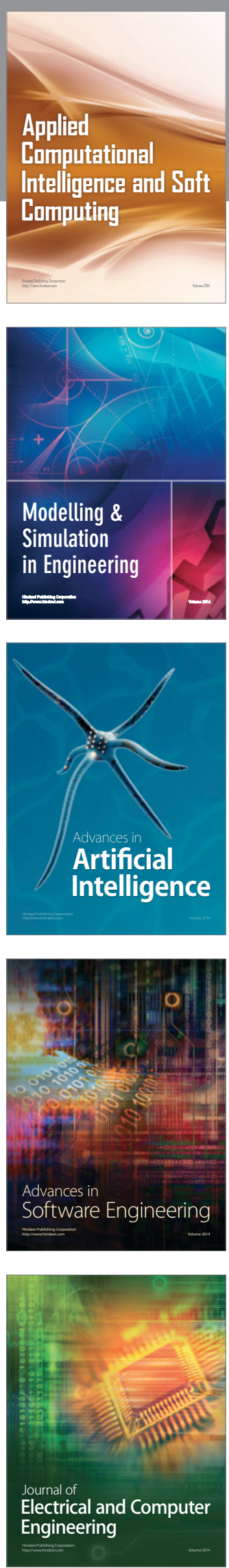